\title{
Low vitamin B12 diet increases liver homocysteine levels and leads to liver steatosis in rats
}

\author{
Imelda Rosalyn Sianipar*, Irena Ujianti**, Sophie Yolanda*, Ahmad Aulia Yusuf***, \\ Neng Tine Kartinah*, Patwa Amani ${ }^{\dagger}$, Krishna Aditya Murti**, ${ }^{\dagger}$ \\ and Dewi Irawati Soeria Santoso*a
}

\begin{abstract}
\section{BACKGROUND}

Nonalcoholic fatty liver disease (NAFLD) is one of the most widespread chronic liver diseases, caused by the development of insulin resistance. One of the mechanisms involved is a disturbance in insulin signaling by certain toxic substances that interact with one of the proteins responsible for the insulin signaling pathway. Increased homocysteine level, upon disruption of the methionine pathway, is associated with insulin resistance. The aim of this study was to evaluate the effect of hyperhomocysteinemia and insulin resistance (HOMA-IR level) induced by dietary vitamin B12 restriction on liver steatosis.

\section{METHODS}

A study of laboratory experimental design was conducted involving 18 male Sprague Dawley rats (age 36-40 weeks, BW 300-350 g), that were randomly divided into 3 groups: control, 8-week treatment, and 16-week treatment. Standard AIN-93 diet was administered to the control group, whereas rats in the treatment groups were fed vitamin B12 deficiency-AIN93M. At the end of treatment, liver homocysteine levels were determined by ELISA, HOMA-IR values were calculated, and steatosis degree of the liver was determined histologically. Statistical analysis was performed using independent t-test.
\end{abstract}

\section{RESULTS}

A significant increase in liver homocysteine levels was found between the control and both the 8 - and 16-week treatment groups $(\mathrm{p}<0.001)$. HOMAIR levels were significantly higher in both treatment groups compared to controls $(\mathrm{p}<0.001)$. The area of liver steatosis in both treatment groups was significantly larger than that of the control group $(\mathrm{p}<0.001)$.

\section{CONCLUSION}

Increased homocysteine levels due to dietary vitamin B12 deficiency induces liver steatosis due to insulin resistance in rats.

Keywords: Homocysteine, vitamin B12 deficiency, NAFLD, insulin resistance, rats
*Department of Medical Physiology,

Faculty of Medicine, Universitas

Indonesia

**Master Program in Biomedical

Science, Faculty of Medicine,

Universitas Indonesia

***Department of Histology,

Faculty of Medicine, Universitas

Indonesia

Department of Physiology,

Faculty of Medicine

UniversitasTrisakti

*Department of Medical Physiology,

Faculty of Medicine University of Indonesia

\section{Correspondence:}

Dewi Irawati Soeria Santoso

Department of Medical Physiology, Faculty of Medicine University of Indonesia

email: dewi.irawati.1307@gmail.com ORCID ID: https://orcid.org/00000001-7390-5298

Date of first submission, December 26, 2018

Date of final revised submission,

December 3, 2019

Date of acceptance, December 11, 2019

This open access article is distributed under a Creative Commons AttributionNon Commercial-Share Alike 4.0 International License

Cite this article as: Sianipar IR, Ujianti I, Yolanda S, et al. Low vitamin B12 diet increases liver homocysteine levels and leads to liver steatosis in rats. UnivMed 2019;38:194-201. doi: 10.18051/ UnivMed.2019.v38.194-201 


\section{INTRODUCTION}

Nonalcoholic fatty liver disease (NAFLD) is defined as the presence of liver steatosis, as demonstrated using imaging or histology, in patients who ingest less than $20 \mathrm{mg}$ of alcohol per day. It is the most prevalent liver disease worldwide, with an estimated global incidence of $25.24 \%$. $^{(1,2)}$ Different metabolic co-morbidities are often associated with NAFLD, including central obesity, insulin resistance, type 2 diabetes mellitus, hyperlipidemia, hypertension, and metabolic syndrome. ${ }^{(3)}$ Nonalcoholic fatty liver disease is an umbrella term which includes a wide spectrum of disorders, ranging from a simple form of steatosis to a progressive form known as nonalcoholic steatohepatitis (NASH), which is characterized by hepatocellular injury and inflammation. Furthermore, NAFLD is considered to be an important cause of cryptogenic cirrhosis. ${ }^{(4)}$

Vitamin deficiency/hypovitaminosis is a general term for a widespread condition which has reached epidemic proportions in developing and developed countries alike, mainly due to lifestyle and dietary habits. Although subclinical hypovitaminosis can occur in well-nourished individuals, it is most often the result of limited dietary intake or vitamin malabsorption. Several studies have shown that chronic liver diseases are associated with lowered levels of vitamins such as vitamin D, E, B6, and A. ${ }^{(5,6)}$ Despite the indications in the literature regarding the association between chronic liver diseases and hypovitaminosis, little is known about any specific correlation between hypovitaminosis and NAFLD.

Vitamin B12 (cobalamin) is a water-soluble cobalt-containing vitamin with an important role in biochemical processes referred to as single carbon transfers. ${ }^{(7)}$ Vitamin B12 is a cofactor for at least 3 enzymes that catalyze these reactions, and acts as a transitional carrier of a single carbon group. It has been reported that several liver diseases such as acute hepatitis, cirrhosis, hepatocellular carcinoma, and metastatic liver disease are associated with major changes in plasma vitamin B12 concentrations. ${ }^{(8)}$ Although these pathologies can lead to alterations in plasma levels of vitamin B12 via different pathways, no clear effect of NAFLD has been determined.

Several studies have shown the correlation between vitamin B12 deficiency and the incidence of steatosis. ${ }^{(1,7,8)}$ A study conducted by Mahamid et al. ${ }^{(9)}$ demonstrated a correlation between low levels of folate and vitamin B12 with the condition of NASH seen histologically. Li et al. ${ }^{(10)}$ reported elevated serum homocysteine in cases of NAFLD in animals induced by high-fat diet, due to decreased transsulfuration pathway regulation.

Several studies have reported that there might be an association between insulin resistance and levels of vitamin B12 and NAFLD. ${ }^{(9-11)}$ This might be explained by the multiple hit theory which states that insulin resistance is the primary basic pathology in liver steatosis. ${ }^{(3)}$ However, the mechanism of vitamin B12 deficiency resulting in liver steatosis via the insulin resistance pathway correlated with increased plasma and liver homocysteine levels is still not widely reported, thus the objective of this study was to investigate the potential correlation between vitamin B12 serum levels and NAFLD, with focus on the insulin resistance pathway as one of the underlying mechanisms.

\section{METHODS}

\section{Study design}

This study was an in vivo experimental study and part of a larger research project at the Department of Medical Physiology Faculty of Medicine Universitas Indonesia. This study was conducted at the Health Research and Development Center (Pusat Penelitian dan Pengembangan Kesehatan, PUSLITBANGKES) Faculty of Medicine Universitas Indonesia (FMUI) from October 2017 to May 2018.

\section{Animals}

This study used male Sprague Dawley rats aged 36-40 weeks with body weight ranging from 
300-350 grams, which were obtained from Badan Penelitian dan Kesehatan RI. Sample size was calculated using Federer's formula, yielding a total of 18 rats. ${ }^{(12)}$

Rats were placed in individual cages in a room with adequate ventilation. Room temperature was maintained between $18-26^{\circ} \mathrm{C}$ with humidity $30-70 \%$ and a 12 -hour light and dark cycle. Rat cages were cleaned every day to ensure maintenance of healthy rats. After one week of acclimatization, they were randomly divided into three groups. The first group was given an AIN-93M diet (Research Diets Inc., USA) ad libitum and was considered as the control group (Group 1, $\mathrm{n}=6$, designated as $\mathrm{C}$ ). The second group was given a modified AIN93M diet ad libitum (AIN-93M with restricted vitamin B12) with additional pectin/cellulose as a source of fiber for eight weeks (Group 2, $\mathrm{n}=6$, designated as T8). The third group was given the same modified AIN-93M diet ad libitum for 16 weeks (Group 3, $\mathrm{n}=6$, designated as $\mathrm{T} 16$ ). The vitamin B12 content in the modified diet was much lower than that in the control diet $(0.016$ vs. $28 \mathrm{mcg} / \mathrm{kg}$ diet). The modified diet also contained $50 \mathrm{~g}$ pectin $/ \mathrm{kg}$ to bind to the intrinsic factor in the intestine and to reduce the bioavailability of vitamin B12. Food intake was recorded daily, and body weight was measured once weekly. After eight and 16 weeks of feeding, levels of plasma vitamin B12, homocysteine and glucose were measured.

\section{Biochemical parameters}

At the end of eight and 16 weeks of diet administration, blood was collected from the supraorbital sinus, after overnight fasting. Liver homocysteine levels were determined using a standard ELISA kit (Rat homocysteine ELISA, MyBiosource, USA). Liver homocysteine assays were performed at the Immuno-Endocrinology Laboratory, Faculty of Medicine, Universitas Indonesia. Insulin resistance indicator values, namely HOMA-IR (homeostasis model assessment values for insulin resistance) were calculated using the formula:
HOMA-IR $=$ fasting glucose $(\mathrm{mmol} / \mathrm{L}) \times$ fasting insulin $(\mu \mathrm{IU} / \mathrm{L}) / 22.5 .^{(13,14)}$

\section{Histological analysis of the liver}

After eight and sixteen weeks of intervention respectively, rats were sacrificed and their livers were fixed in $10 \%$ buffered formalin and embedded in paraffin. Liver sections $(5 \mu \mathrm{m})$ were stained with hematoxylin and eosin (H\&E), and the steatotic degrees of individual liver samples were determined by subjecting sections to blind examination by a pathologist; sections were scored depending on the percentage of hepatocytes containing lipid droplets. ${ }^{(15,16)}$ Liver histology preparations were carried out at the Histology Laboratory FMUI.

\section{Ethical clearance}

Rats were treated in accordance with the Helsinki convention. Ethical approval was obtained from the Health Research Ethics Committee - Faculty of Medicine Universitas Indonesia/Cipto Mangunkusumo Hospital (FKUI/ RSCM) under number: 184/UN2.F1/ETIK/2017.

\section{Statistical analysis}

Statistical analysis was performed using oneway ANOVA followed by post-hoc analysis for homocysteine levels and liver steatosis, while independent $\mathrm{t}$-test and paired t-test were used for HOMA-IR, in which the treatment group was compared to the control group in their respective treatment week, and the HOMA-IR value was also assessed between treatment groups. Data were previously analyzed for normality using the Shapiro-Wilk test. Data processing was performed using the SPSS 12 software (Statistical Social Sciences program version 12).

\section{RESULTS}

\section{Liver homocysteine levels}

Based on the one-way ANOVA test, group T16 showed significantly higher levels of liver homocysteine $(\mathrm{p}<0.001)$ compared to both control and T8 groups (Table 1). 
Table 1. Homocysteine levels and percent of steatosis from liver tissue and plasma HOM-IR by treatment groups

\begin{tabular}{|c|c|c|c|c|}
\hline & \multicolumn{2}{|c|}{$\begin{array}{c}\mathrm{C} \\
(\mathrm{n}=6)\end{array}$} & $\begin{array}{c}\mathrm{T} 8 \\
(\mathrm{n}=6)\end{array}$ & $\begin{array}{c}\mathrm{T} 16 \\
(\mathrm{n}=6)\end{array}$ \\
\hline Liver homocysteine ${ }^{@}$ & \multicolumn{2}{|c|}{$1.52 \pm 0.05$} & $1.6 \pm 0.05$ & $2.48 \pm 0.20^{* * * \# \#}$ \\
\hline$\%$ of steatosi ${ }^{@}$ & \multicolumn{2}{|c|}{$3.33 \pm 0.25$} & $53.83 \pm 4.56^{* * *}$ & 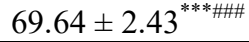 \\
\hline & $\mathrm{C} 8$ & T8 & $\mathrm{C} 16$ & $\mathrm{~T} 16$ \\
\hline $\mathrm{HOMA}-\mathrm{IR}^{\wedge}(\mu \mathrm{IU} / \mathrm{L}) / 22.5$ & $2.27 \pm 0.07$ & $3.91 \pm 0.13^{* *}$ & $3.03 \pm 0.05$ & $5.87 \pm 0.09$ \\
\hline
\end{tabular}

Values are expressed as Mean $\pm \mathrm{SD}$; Dietary groups: C: Control; C8: Control at week 8;T8: AIN-93M with restricted vitamin B12 for 8 weeks, C16: Control a week 16;T16: AIN-93M with restrided vitamin B12 for 16 weks; ; to control; ${ }^{\# \#} \mathrm{p}<0.01$ compared to T8; ${ }^{\circledR}$ One-way ANOVA; ${ }^{\wedge}$ Independent and paired t-tests

\section{HOMA-IR values}

Based on the independent t-test, there was a significant difference in HOMA-IR values between treatment groups $\mathrm{T} 8$ and $\mathrm{T} 16(\mathrm{p}<0.001)$, with group T16 showing higher HOMA-IR values. Based on the paired t-test, there were significant differences in HOMA-IR values between control and treatment groups in both week 8 and week 16 (Table 1).

\section{Liver histopathology}

There was no obvious steatosis in the livers of the control group. The area of hepatic steatosis in both treatment groups were significantly larger compared to controls $(\mathrm{p}<0.001)$, with the largest area of hepatic steatosis found in group T16 $(\mathrm{p}<0.001$ vs T8) (Table 1$)$.

\section{DISCUSSION}

NAFLD is a widespread chronic liver disease and its incidence is increasing in developed countries. Our previous study showed that vitamin B12 restricted diet caused lower vitamin B12 plasma levels. ${ }^{(17)}$ Thie study also showed that the decrease in plasma vitamin B12 levels caused increased plasma homocysteine levels. ${ }^{(17)}$ In this study, there was a significant increase in liver homocysteine levels in the treatment groups, namely approximately twice as high as in the control group. The study supports the use of vitamin B12 deficient rats as an animal model for hyperhomocysteinemia.

In vitamin B12 deficient rats, methionine metabolism is impaired. The methyl group of B12 functions as a coenzyme of methionine synthase which catalyzes the methyl transfer from methyltetrahydrofolate to homocysteine, resulting in the donation of a methyl group to homocysteine, forming methionine. ${ }^{(18)}$ Methionine synthase is important to re-synthesize methionine and to metabolize methyltetrahydrofolate. In patients with vitamin B12 deficiency leading to decreased liver transsulfuration pathways in homocysteine metabolism, an increase in plasma homocysteine level was observed. ${ }^{(19)}$ Increased plasma homocysteine causes oxidative stress, resulting in insulin resistance through the ROS pathway. ${ }^{(20,21)}$

According to the literature, oxidative stress produced by the conversion of homocysteine (Hcy) to Hcy thiolactone leads to the inhibitory activity of the tyrosine kinase insulin receptor. A study by Najib and Sanchez-Margalet (22) showed that homocysteine thiolactone, the active form of homocysteine, inhibited the insulinstimulated tyrosine phosphorylation of insulin receptor $\beta$-subunit and its substrates (insulin receptor substrate-1 and p60-70) in rat hepatoma cells. In addition, they showed that homocysteine thiolactone decreased the $\mathrm{p} 85$ regulatory subunit of phosphatidylinositol 3-kinase activity, inducing a reduction in insulin-stimulated glycogen synthesis. Insulin signaling disorder results in an increase in plasma blood glucose. This is in accordance with our previous study, in that the significantly higher fasting blood glucose levels in rats receiving fed restricted $\mathrm{B} 12$ diet indicated that the restriction of vitamin B12 could lead to the development of insulin resistance. This is 

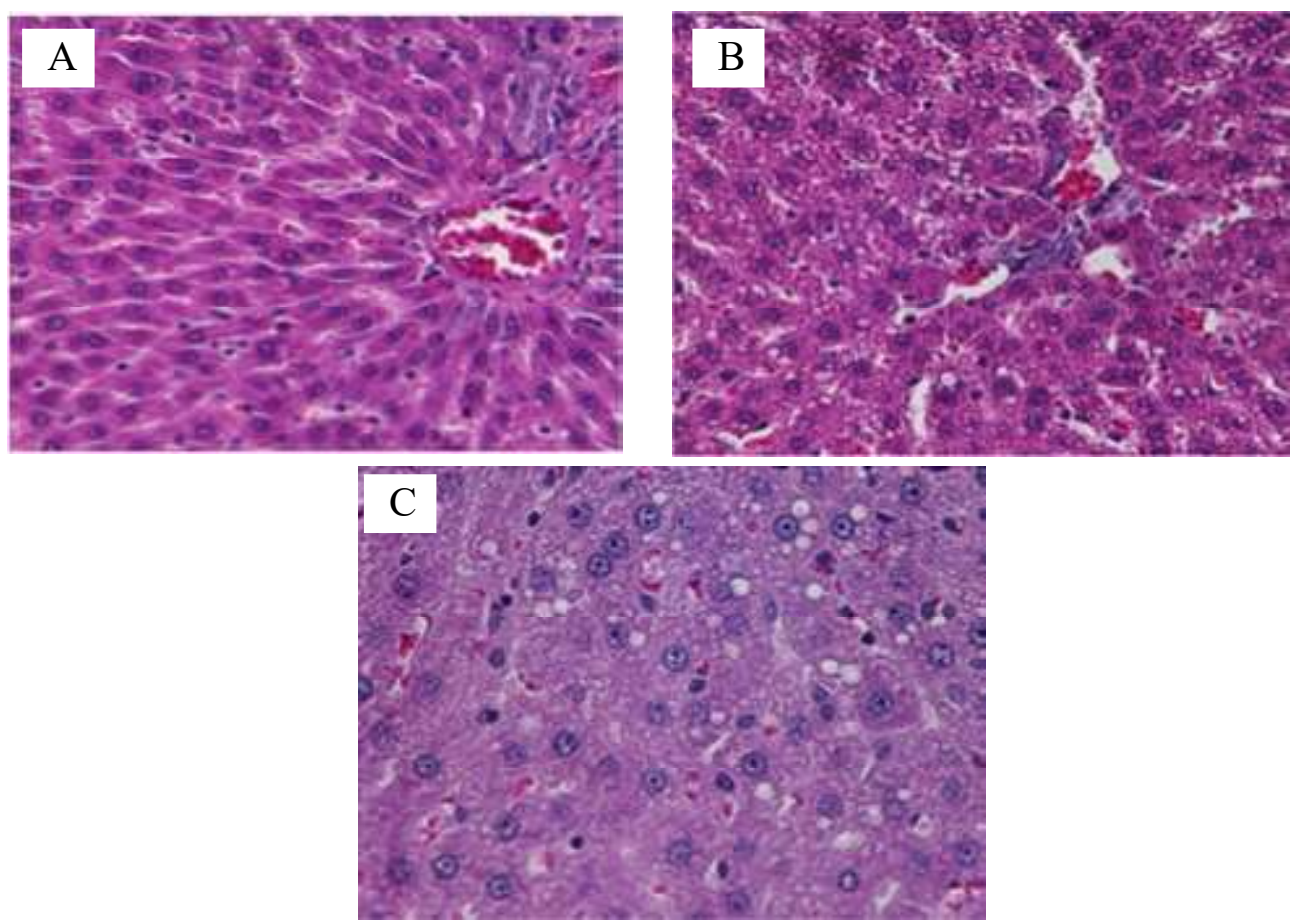

Figure 1. Liver histology of a normal rat (C16) (a) and NAFLD rat at week 8 (T8);

(b) and week 16 (T16); (c) (Magnification 40x)

consistent with several studies that suggest a link between increased homocysteine due to vitamin B12 deficiency and insulin resistance. ${ }^{(11,23)}$ Our study also showed similar results to these studies, which was shown by the HOMA-IR values.

Another study suggested that hepatic insulin resistance can perturb the regulation of lipid metabolism and it is a common feature observed in human and rodent models of NAFLD. ${ }^{(24)}$ The regulation of glucose and lipid production is disrupted when insulin action is compromised in the liver. A study by Abdul-Ghani and De Fronzo (25) have documented $35-50 \%$ reductions in glucose disposal, a measure of whole-body insulin sensitivity, and an impaired ability of insulin to suppress endogenous glucose production, indicative of hepatic insulin resistance Additionally, subjects with NAFLD exhibit a defect in insulin suppression of free fatty acids (FFA), in keeping with insulin resistance at the level of the adipocyte. ${ }^{(26)}$ Compared with controls, subjects with NAFLD also demonstrate a blunted inhibition of fatty acid oxidation, reflecting the decreased uptake and use of glucose as a source of fuel. These findings suggest the possibility that insulin resistance may be an intrinsic defect in NAFLD, in which diminished insulin responsiveness at the level of the adipocyte may contribute to hepatic steatosis by excess FFA flux to the liver. ${ }^{(27)}$

Expression of genes involved in the de novo lipogenesis process in the liver is also significantly increased in NAFLD patients. In healthy conditions, lipogenesis contributed 5\% of the total amount of liver triglycerides. But this number is increased by $26 \%$ in patients with NAFLD. This biosynthetic increase contributed greatly to NAFLD patients. ${ }^{(28)}$ Although there was an increase in regulation of fatty acid oxidation and export of triglycerides by VLDL in NAFLD patients, this process cannot restore homeostasis in the liver. ${ }^{(29)}$

Besides via insulin resistance, vitamin B12 deficiency contributes to the development of steatosis through hyperhomocysteinemia. Vitamin B12 is a very important molecule for the process of synthesizing S-adenosylmethionine (SAM) 
from methionine. Phosphatidylethanolamine Nmethyltransferase (PEMT) uses SAM as a methyl donor to catalyze methylation of phosphatidylethanolamine (PE) to phosphatidylcholine (PC), which is needed for VLDL formation in hepatocytes. Phosphatidylcholine synthesis decreases in vitamin B12 deficiency. Phosphatidylcholine affects lipid export by VLDL, and causes accumulation of liver lipids. ${ }^{\left({ }^{8,30}\right)}$ In addition, disorders of homocysteine metabolism due to vitamin B12 deficiency cause a decrease in the transsulfuration pathway in the liver, increase the SAM/SAH comparison index causing liver steatosis. ${ }^{(31)}$

Due to an increase in the prevalence of risk factors for vitamin B12 deficiency, such as advanced age, vegetarian lifestyle, or patients with gastrointestinal problems which result in impaired absorption of vitamin B12, one of the consequences of these conditions is liver steatosis. ${ }^{(32)}$ This study explores one of the pathways of liver steatosis due to vitamin B12 deficiency via the insulin resistance pathway. It is hoped that the results of this study may help in preventing the adverse effects of vitamin B12 deficiency in high-risk populations.

One of the limitations of this study is that although adverse effects of vitamin B12 deficiency have been observed, the rats were clinically not yet categorized as having vitamin B12 deficiency. It is hoped that future studies will address this issue through modification of the length of the feeding or an increase in the percentage of pectin which can lead to vitamin B12 deficiency.

\section{CONCLUSION}

This study demonstrated that vitamin B12 restricted diet results in the occurrence of liver steatosis via insulin resistance and hyperhomocysteinemia in rats. And elevated homocysteine was a critical factor in the pathogenesis of NAFLD.

\section{ACKNOWLEDGEMENT}

This study was funded by a grant provided by the Ministry of Research, Technology and Higher Education, Republic of Indonesia, HIBAH Pasca Sarjana 2018 with contract number 551/UN2.R3.1/HKP05.00/2018.

\section{CONFLICT OF INTEREST}

The authors declare no conflict of interest.

\section{CONTRIBUTORS}

DISS and IRS contributed to the concept and design. IU and IRS contributed to writing the original draft. IU and SY contributed to literature search. DISS and IRS contributed to the investigation. AAY, PA and KAM contributed to data collection and analysis. IRS, DISS, and contributed to writing the revision and editing. All authors have read and approved the final manuscript.

\section{REFERENCES}

1. Lindenmeyer CC, McCullough AJ. The natural history of nonalcoholic fatty liver disease: an evolving view. Clin Liver Dis 2018;22:11-21. doi: 10.1016/j.cld.2017.08.003

2. Abd El-Kader SM, El-Den Ashmawy EMS. Nonalcoholic fatty liver disease: the diagnosis and management. World J Hepatol 2015;7:846-58. doi: 10.4254/wjh.v7.i6.846.

3. Buzzetti E, Pinzani M, Tsochatzis EA. The multiplehit pathogenesis of non-alcoholic fatty liver disease (NAFLD). Metabolism 2016;65:1038-48. doi: 10.1016/j.metabol.2015.12.012.

4. Takahashi Y, Fukusato T. Histopathology of nonalcoholic fatty liver disease/nonalcoholic steatohepatitis. World J Gastroenterol 2014;20: 15539-48. doi: 10.3748/wjg.v20.i42.15539.

5. Barchetta I, Cimini FA, Cavallo MG. Vitamin D supplementation and non-alcoholic fatty liver disease: present and future. Nutrients 2017;9:pii: E1015. doi: 10.3390/nu9091015.

6. Bito T, Misaki T, Yabuta Y, Ishikawa T, Kawano T, Watanabe F. Vitamin B12 deficiency results in severe oxidative stress, leading to memory 
retention impairment in Caenorhabditis elegans. Redox Biol 2017;11:21-9. doi: 10.1016/j.redox.2016. 10.013 .

7. Koplay M, Gulcan E, Ozkan F. Association between serum vitamin B12 levels and the degree of steatosis in patients with nonalcoholic fatty liver disease. J Investig Med 2011;59:1137-40. doi: 10.2310/JIM.0b013e31822a29f5.

8. Huwait EA, Kumosani TA, Moselhy SS, Mosaoa RM, Yaghmoor SS. Relationship between soil cobalt and vitamin B12 levels in the liver of livestock in Saudi Arabia: role of competing elements in soils. Afr Health Sci 2015;15:993-1008. doi: 10.4314/ahs.v15i3.38.

9. Mahamid M, Mahroum N, Bragazzi NL, et al. Folate and B12 levels correlate with histological severity in NASH patients. Nutrients 2018;10:440. doi: 10.3390/nu10040440.

10. Li Z, Gueant-Rodriguez R, Quilliot D, et al. Folate and vitamin B12 status is associated with insulin resistance and metabolic syndrome in morbid obesity. Clin Nutr 2018;37:1700-6. doi: 10.1016/ j.clnu.2017.07.008.

11. Yang N, Yao Z, Miao L, et al. Novel clinical evidence of an association between homocysteine and insulin resistance in patients with hypothyroidism or subclinical hypothyroidism. PLoS One 2015;10:e0125922. doi: 10.1371/journal.pone.0125922.

12. Charan J, Kantharia ND. How to calculate sample size in animal studies/? J Pharmacol Pharmacother 2013;4:303-6. doi: 10.4103/0976-500X.1197262013.

13. Andraini T, Yolanda S. Prevention of insulin resistance with Hibiscus sabdariffa Linn. extract in high-fructose fed rat. Med J Indones 2015;23:192-6. http://dx.doi.org/10.13181/ mji.v23i4.848.

14. Antuna-Puente B, Disse E, Rabasa-Lhoret R, Laville M, Capeau J, Bastard JP. How can we measure insulin sensitivity/resistance/? Diabetes Metab 2011;37:179-88. doi: 10.1016/j.diabet.2011. 01.002 .

15. Liang W, Menke AL, Driessen A, et al. Establishment of a general NAFLD scoring system for rodent models and comparison to human liver pathology. PLoS One 2014;9:e115922. doi: 10.1371/journal.pone.0115922.

16. Rastogi A, Shasthry SM, Agarwal A, et al. Nonalcoholic fatty liver disease - histological scoring systems: a large cohort single-center, evaluation study. APMIS 2017;125:962-73. doi: 10.1111/ apm.12742.2017;962-73.

17. Sianipar IR, Ujianti I, Yolanda S, et al. Developing vitamin B12 deficient rat model based on duration of restriction diet: assessment of plasma vitamin
B12, homocysteine (Hcy), and blood glucose levels. In: Wulan PPDK, Gozan M, Astutiningsih S, Ramahdita G, Dhelika R, Kreshanti P, editors. $3^{\text {rd }}$ Biomedical Engineering"s Recent Progress in Biomaterials, Drugs Development, and Medical Devices. Proceedings of the International Symposium of Biomedical Engineering, ISBE; 2018 Aug 6-8; American Institute of Physics Inc.;2019.p.10001.

18. Mahmood L. The metabolic processes of folic acid and vitamin B12 deficiency. J Health Res Rev 2014;1:5-9. doi: 10.4103/2394-2010.143318.

19. Mahalle N, Kulkarni MV, Garg MK, Naik SS. Vitamin B12 deficiency and hyperhomocysteinemia as correlates of cardiovascular risk factors in Indian subjects with coronary artery disease. J Cardiol 2013;61:28994. doi: 10.1016/j.jjcc.2012.11.009.

20. Feng $\mathrm{X}, \mathrm{Xu}$ Y. Hyperhomocysteinemia as a metabolic risk factor for glucose intolerance among high-risk groups of Chinese adults. Med Sci Monit 2017;2775-81. doi: 10.12659/msm. 905002.

21. Platt DE, Hariri E, Salameh P, et al. Type II diabetes mellitus and hyperhomocysteinemia: a complex interaction. Diabetol Metab Syndr 2017;9:19. doi: 10.1186/s13098-017-0218-0.

22. Najib S, Sánchez-Margalet V. Homocysteine thiolactone inhibits insulin-stimulated DNA and protein synthesis: possible role of mitogenactivated protein kinase (MAPK), glycogen synthase kinase-3 (GSK-3) and p70 S6K phosphorylation. J Mol Endocrinol 2005;34:11926. doi: 10.1677/jme.1.01581.

23. Ebrahimpour A, Vaghari-tabari M, Qujeq D, Moein S. Direct correlation between serum homocysteine level and insulin resistance index in patients with subclinical hypothyroidism: does subclinical hypothyroidism increase the risk of diabetes and cardio vascular disease together/? Diabetes Metab Syndr 2018;12:863-7. doi: 10.1016/ j.dsx.2018.05.002.

24. Zheng S, Xu H, Zhou H, et al. Associations of lipid profiles with insulin resistance and $\beta$ cell function in adults with normal glucose tolerance and different categories of impaired glucose regulation. PLoS One 2017;12:e0172221. doi: 10.1371/journal.pone.0172221.2017;1-11.

25. Birkenfeld AL, Shulman GI. Nonalcoholic fatty liver disease, hepatic insulin resistance, and type 2 diabetes. Hepatology 2014;59:713-23. doi: 10.1002/hep.26672.

26. Abdul-Ghani MA, De Fronzo RA. Pathogenesis of insulin resistance in skeletal muscle. J Biomed 
Biotechnol 2010, Article ID 476279, 19 pages. doi: $10.1155 / 2010 / 476279$.

26. Cook JR, Langlet F, Kido Y, Accili D. Pathogenesis of selective insulin resistance in isolated hepatocytes. J Biol Chem 2015;290:13972-80. doi: 10.1074/jbc.M115.638197.

27. Flegal KM, Carroll MD, Ogden CL, Curtin LR. Prevalence and trends in obesity among US adults, 1999-2008. JAm Med Assoc 2010;303:23541. doi: 10.1001/jama.2009.2014.

28. Perry RJ, Samuel VT, Petersen KF, Shulman GI. Insulin resistance and type 2 diabetes. Nature 2014;510:84-91. doi: 10.1038/nature13478.

29. Lambert JE, Ramos-Roman MA, Browning JD, Parks EJ. Increased de novo lipogenesis is a distinct characteristic of individuals with nonalcoholic fatty liver disease. Gastroenterology
2014;146:726-35. http://dx.doi.org/10.1053/ j.gastro.2013.11.049.

30. Gluchowski NL, Becuwe M, Walther TC, Farese Jr RV. Lipid droplets and liver disease: from basic biology to clinical implications. Nat Rev Gastroenterol Hepatol 2017;14:343-55. doi: 10.1038/nrgastro.2017.32.

31. Rolo AP, Teodoro JS, Palmeira CM. Role of oxidative stress in the pathogenesis of nonalcoholic steatohepatitis. Free Radic Biol Med 2012;52:59-69. doi: 10.1016/j.freeradbiomed.2011. 10.003.

32. Peverill W, Powell LW, Skoien R. Evolving concepts in the pathogenesis of NASH: beyond steatosis and inflammation. Int $\mathrm{J}$ Mol Sci 2014;15:8591-638. doi: 10.3390/ijms15058591. 